(Supporting Information)

\title{
A Refined Empirical Force Field to Model Protein-SAM Interactions Based on AMBER14 and GAFF
}

\author{
Pratiti Bhadra and Shirley W.I. Siu* \\ Department of Computer and Information Science, University of Macau, Taipa, Macau \\ E-mail: shirleysiu@umac.mo
}

Phone: +853 8822-4452. Fax: $+8538822-2426$ or +8533974285

Number of Pages: 9

Number of Tables: 4

Number of Figures: 6 


\section{Table of Contents}

Table S1. Gaussian potential widths for the eleven host-guest peptides used in WT-MetaD simulations.

Table S2. Theoretical thickness values of the adsorbed layers of host-guest peptides.

Table S3. Summary of the modified parameters for glutamic acid (E) and glutamine (Q) amino acid residues.

Table S4. Lysozyme initial orientations predicted by ProtPOS using standard GAFF and Amber ff14sb parameters.

Figure S1. Histogram from US window simulations of peptide TGTG-F-GTGT.

Figure S2. PMF profiles computed from 6, 8, and 10 ns trajectory of the TGTG-F-GTGT simulation showing trend of convergence.

Figure S3. Snapshots of protein orientations on the CH3-SAM surface using the standard parameters.

Figure S4. Surface separating distance (SSD) profiles of the two protein orientations on the CH3SAM surface.

Figure S5. Snapshots of protein orientations on the CH3-SAM surface at different time frames (40 and $240 \mathrm{~ns}$ ) from Cluster 1 simulation.

Figure S6. Snapshots of protein orientations on the CH3-SAM surface at different time frames (40, 70, 105, and $115 \mathrm{~ns})$ from Cluster 2 simulation. 
Table S1: Gaussian potential widths for the eleven host-guest peptides used in WT-MetaD simulations. The symbol $-\mathrm{X}$ - denotes the guest residue in a host-guest peptide.

\begin{tabular}{c|c|c|c|c|c}
\hline -X- & width (nm) & $-\mathrm{X}-$ & width $(\mathrm{nm})$ & $-\mathrm{X}-$ & width $(\mathrm{nm})$ \\
\hline -L- & 0.06 & $-\mathrm{T}-$ & 0.02 & $-\mathrm{R}-$ & 0.15 \\
-F- & 0.02 & $-\mathrm{G}-$ & 0.02 & $-\mathrm{K}-$ & 0.08 \\
-V- & 0.02 & $-\mathrm{S}-$ & 0.03 & $-\mathrm{D}-$ & 0.03 \\
-W- & 0.02 & $-\mathrm{N}-$ & 0.07 & & \\
\hline
\end{tabular}

Table S2: Theoretical thickness values of the adsorbed layers of host-guest peptides as reported in Ref. ${ }^{1}$

\begin{tabular}{c|ccccccccccc}
\hline$-\mathrm{X}-$ & $-\mathrm{L}-$ & $-\mathrm{F}-$ & $-\mathrm{V}-$ & $-\mathrm{W}-$ & $-\mathrm{T}-$ & $-\mathrm{G}-$ & $-\mathrm{S}-$ & $-\mathrm{N}-$ & $-\mathrm{R}-$ & $-\mathrm{K}-$ & $-\mathrm{D}-$ \\
\hline thickness $(\AA)$ & 12.1 & 12.3 & 12.0 & 12.5 & 12.0 & 11.8 & 12.0 & 12.1 & 12.3 & 12.2 & 12.1 \\
\hline
\end{tabular}


Table S3: Summary of the modified parameters for glutamic acid (E) and glutamine (Q) amino acid residues. These modified parameters have been made based on the similarity of their atom types to aspartic acid (D) and asparagine (N).

\begin{tabular}{|c|c|c|c|c|}
\hline SAM & & Amino Acid & & \\
\hline & $-\mathrm{X}-$ & IUPAC $^{b}$ name & Standard $^{c}$ & $\operatorname{Modified}^{d}\left(\mathrm{O}_{f}\right)^{e}$ \\
\hline$\left(\right.$ atom type $\left.^{a}\right)$ & & $\left(\right.$ atom type $^{c} \rightarrow$ new atom type $\left.{ }^{d}\right)$ & $\epsilon_{i j}$ & $\epsilon_{i j}$ \\
\hline \multirow{5}{*}{ C12 (c3) } & $-\mathrm{Q}-$ & $\mathrm{ND} 2(\mathrm{~N} \rightarrow \mathrm{NF})$ & 0.5706 & $0.8559(1.5)$ \\
\hline & $-Q-$ & HD21, HD22(H $\rightarrow$ HF) & 0.1734 & $0.2601(1.5)$ \\
\hline & $-\mathrm{E}-$ & $\mathrm{OD} 1, \mathrm{OD} 2(\mathrm{O} 2 \rightarrow \mathrm{O} 2 \mathrm{~F})$ & \multirow{2}{*}{0.6342} & \multirow{2}{*}{$0.9513(1.5)$} \\
\hline & $-Q-$ & $\mathrm{OD} 1(\mathrm{O} \rightarrow \mathrm{OF})$ & & \\
\hline & $\begin{array}{l}-\mathrm{E}- \\
-\mathrm{Q}-\end{array}$ & $\mathrm{CG}(\mathrm{C} \rightarrow \mathrm{CF})$ & 0.4058 & $0.9131(2.25)$ \\
\hline \multirow{5}{*}{$\mathrm{H} 12 \mathrm{~A}, \mathrm{H} 12 \mathrm{~B}, \mathrm{H} 12 \mathrm{C}$ (hc) } & $-\mathrm{Q}-$ & $\mathrm{ND} 2(\mathrm{~N} \rightarrow \mathrm{NF})$ & 0.2162 & $0.8646(4)$ \\
\hline & $-\mathrm{Q}-$ & HD21, HD22 (H $\rightarrow$ HF) & 0.0657 & $0.2628(4)$ \\
\hline & $-\mathrm{E}-$ & $\mathrm{OD} 1, \mathrm{OD} 2(\mathrm{O} 2 \rightarrow \mathrm{O} 2 \mathrm{~F})$ & \multirow{2}{*}{0.2402} & \multirow{2}{*}{$0.9610(4)$} \\
\hline & $-\mathrm{Q}-$ & $\mathrm{OD} 1(\mathrm{O} \rightarrow \mathrm{OF})$ & & \\
\hline & $\begin{array}{l}-\mathrm{E}- \\
-\mathrm{Q}-\end{array}$ & $\mathrm{CG}(\mathrm{C} \rightarrow \mathrm{CF})$ & 0.1537 & $0.9924(6.5)$ \\
\hline
\end{tabular}

${ }^{a}$ GAFF atom type

${ }^{b}$ IUPAC: International union of pure and applied chemistry

${ }^{c}$ AMBER ff14SB atom type and interaction parameter

${ }^{d}$ New atom type with modified interaction parameter

${ }^{e} \mathrm{O}_{f}$ : optimization factor; see Method section in main text. 
Table S4: Lysozyme initial orientations predicted by ProtPOS using standard GAFF and Amber ff14sb parameters.

\begin{tabular}{c|c|c}
\hline & $\begin{array}{c}\text { ProtPOS score } \\
(\mathrm{kcal} / \mathrm{mol})\end{array}$ & $\begin{array}{c}\text { Predicted initial } \\
\text { adsorption orientation }\end{array}$ \\
\hline Cluster 1 $(37.5 \%)$ & -101.6 & $\begin{array}{c}\alpha=18.92^{\circ}, \omega= \\
139.63^{\circ} \text { (side-on, } \\
\text { face-down) }\end{array}$ \\
\hline Cluster 2(62.5\%) & -90.6 & $\begin{array}{c}\alpha=18.68^{\circ}, \omega=96.37^{\circ} \\
\text { (side-on, face-down) }\end{array}$ \\
\hline
\end{tabular}

${ }^{a}$ Orientation of lysozyme with respect to the surface is referred as "side-on" ( $\alpha$ close to $0^{\circ}$, where $\alpha$ is the angle between the long axis of the protein and the surface plane) or "end-on" ( $\alpha$ close to $90^{\circ}$ ); and the orientation of protein's active site is referred as "face-up" ( $\omega$ is less than $90^{\circ}$, where $\omega$ is the angle between the surface normal and the vector from the protein center to the active site) or "face-down" ( $\omega$ is greater than $90^{\circ}$ ). The long axis of the protein was taken as the vector of Asn46-C $\alpha$ to Ala9-C $\alpha$; the center of mass of two catalytic residues, Glu35 and Asp52, were used to define the location of the active site. 


\section{Umbrella histograms}

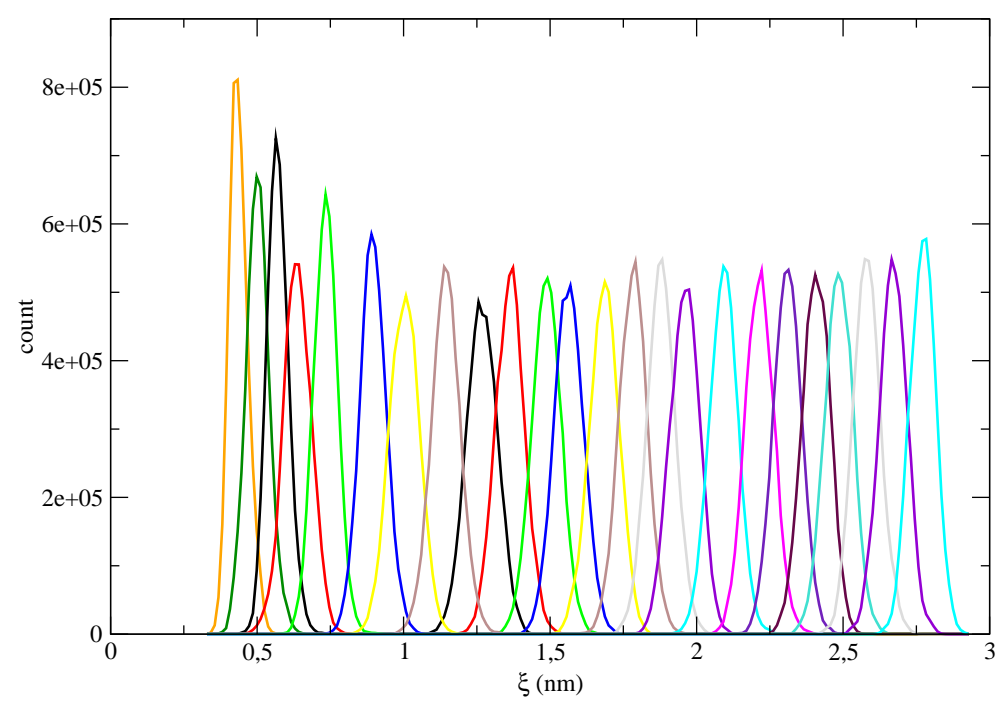

Figure S1: Histograms from US window simulations of peptide TGTG-F-GTGT. Parameters used: $1 \AA$ window interval, $\mathrm{k}=1000 \mathrm{~kJ} \mathrm{~mol}^{-1} \mathrm{~nm}^{-2}$, and $10 \mathrm{~ns}$ simulation time.

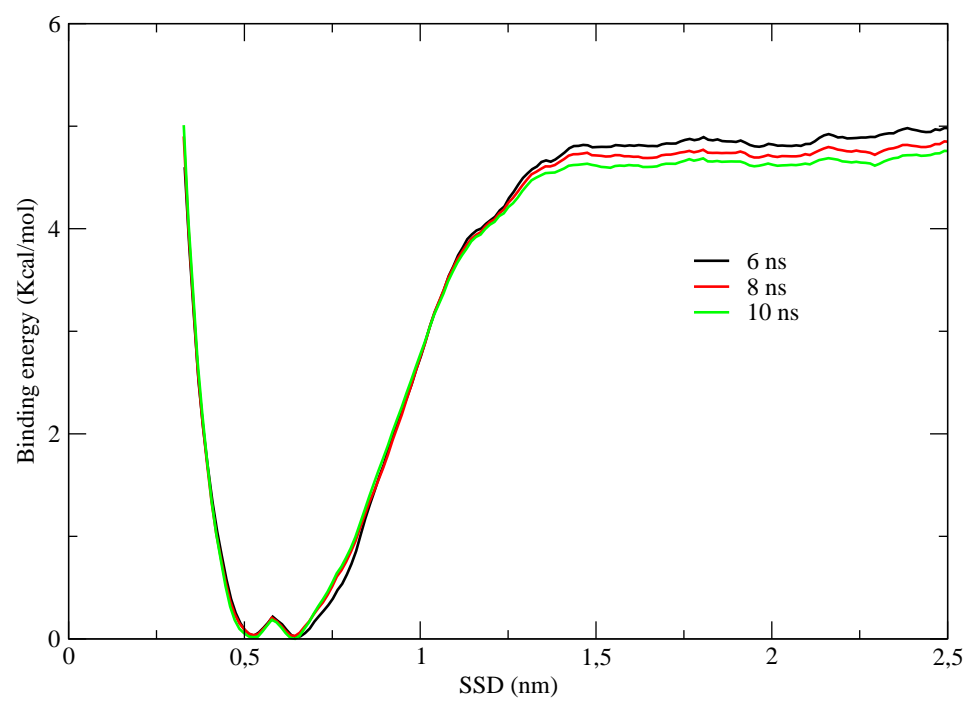

Figure S2: PMF profiles computed from 6, 8, and 10 ns trajectory of the TGTG-F-GTGT simulation showing trend of convergence. 

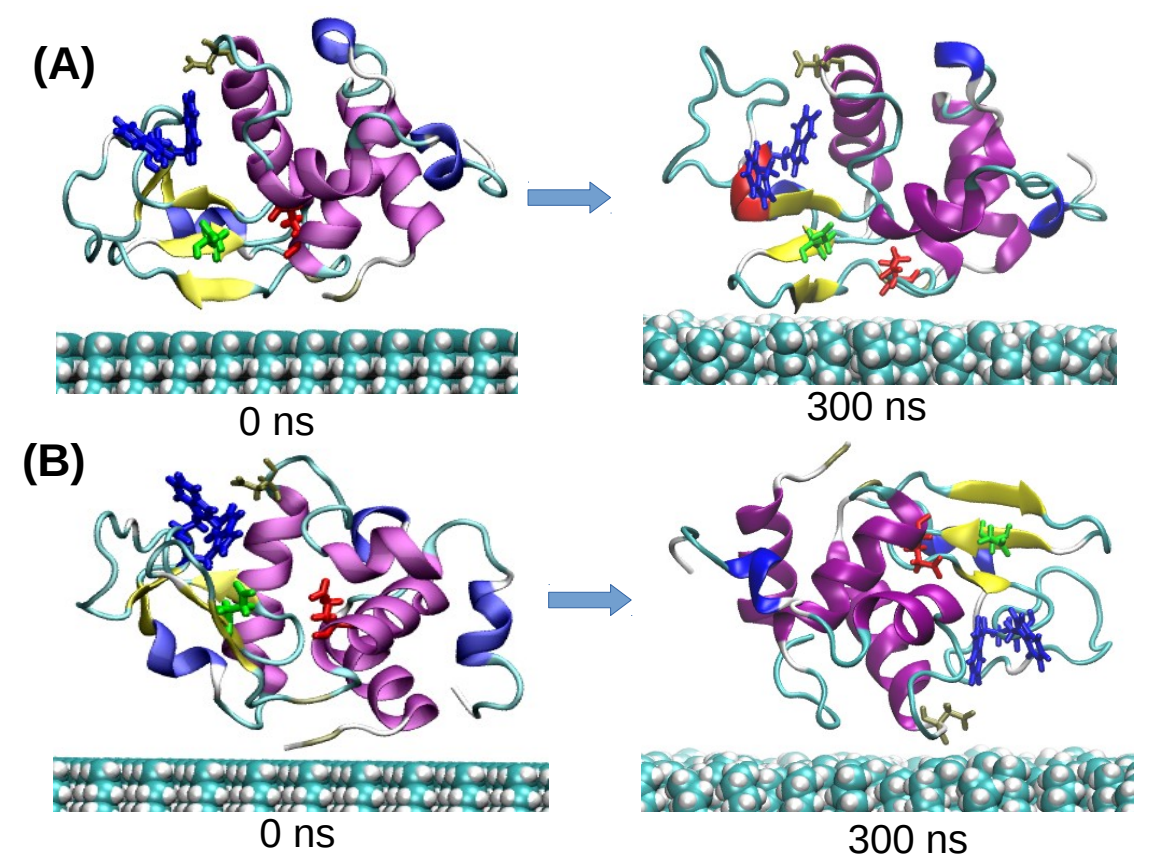

Figure S3: Snapshots of protein orientations on the CH3-SAM surface using the standard parameters: (A) Cluster 1 (face-down) and (B) Cluster 2 (face-down). Initial structures were predicted by ProtPOS using the standard parameters. The protein is shown in cartoon style with secondary structure coloring. Important residues at the active site are shown using the stick model and colored: Glu 35 (red) and Asp 52 (green), Trp 62 (blue) and Trp 63 (blue)
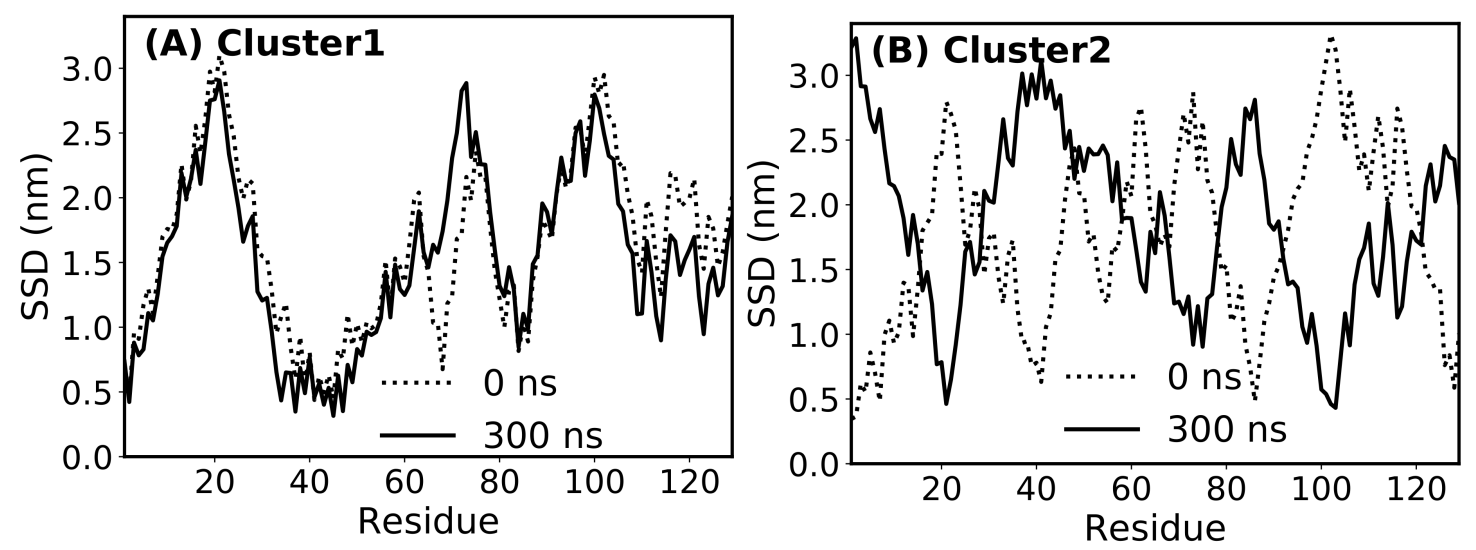

Figure S4: Surface separating distance (SSD) profiles of the two protein orientations on the $\mathrm{CH}_{3}$ SAM surface (see Figure S3). 

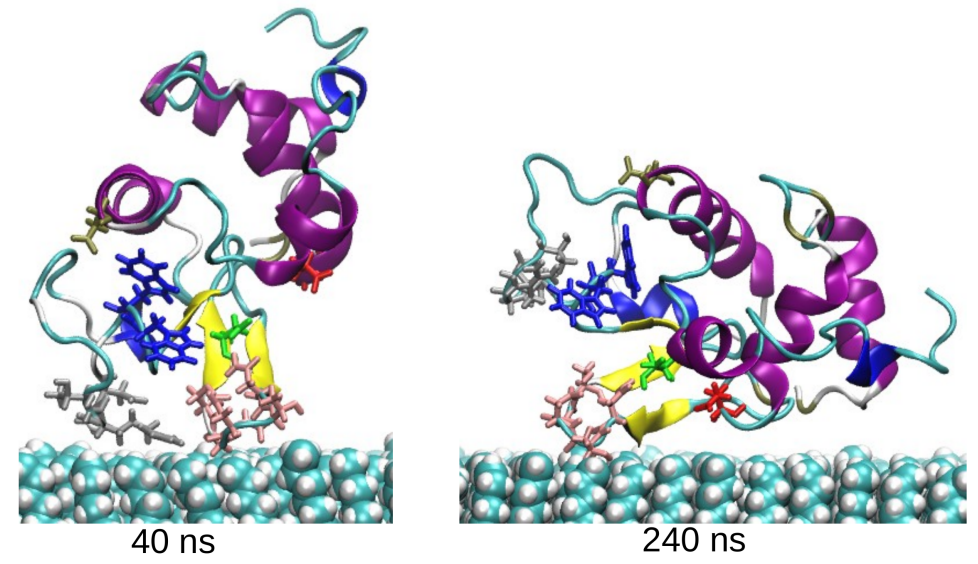

Figure S5: Snapshots of protein orientations on the CH3-SAM surface at different time frames (40 and $240 \mathrm{~ns}$ ) from Cluster 1 simulation. The protein is shown in cartoon style with secondary structure coloring. Important residues at the active site are shown using the stick model and colored: Glu 35 (red) and Asp 52 (green); Trp 62 (blue) and Trp 63 (blue). Transient contact residues with the surface are highlighted: Asp66-Pro70 (pink); Asn46-Ser50 (silver)
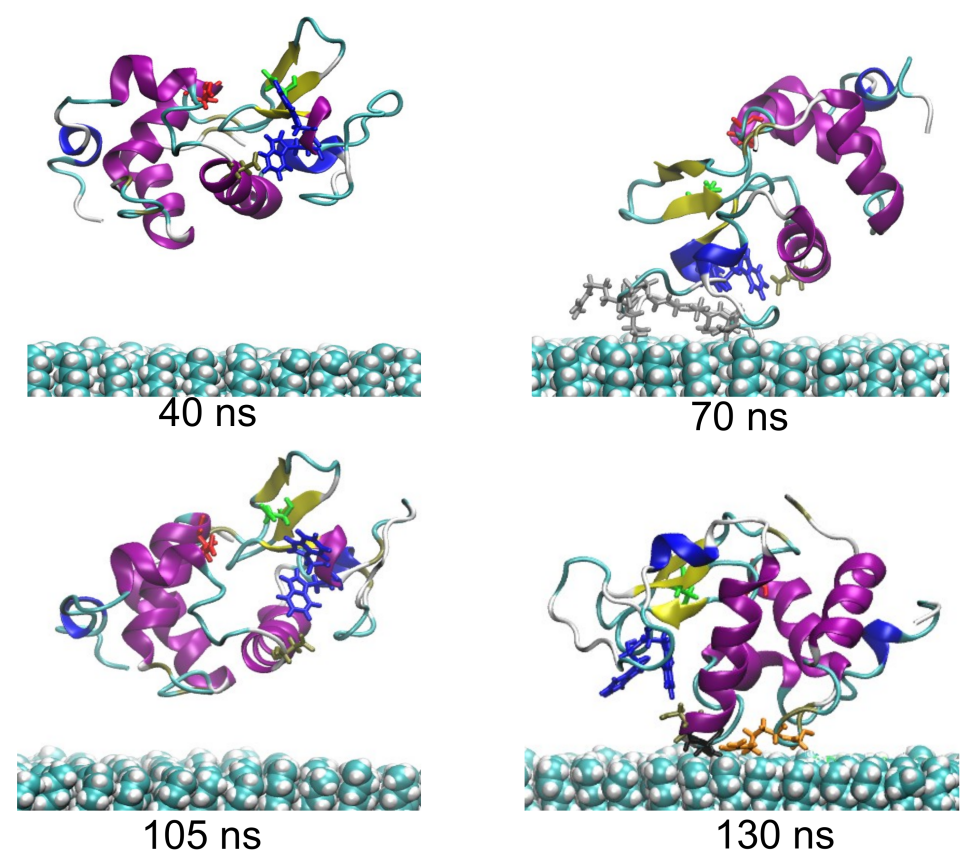

Figure S6: Snapshots of protein orientations on the CH3-SAM surface at different time frames (40, 70, 105 and $115 \mathrm{~ns}$ ) from Cluster 2 simulation. The protein is shown in cartoon style with secondary structure coloring. Important residues at the active site are shown using the stick model and colored: Glu 35 (red) and Asp 52 (green); Trp 62 (blue) and Trp 63 (blue). Transient contact residues with the surface are highlighted: Gly67-Asn74 (silver); Arg21-Gly22 (black); Gly102Asn103 (orange). 


\section{References}

(1) Wei, Y.; Latour, R. A. Benchmark Experimental Data Set and Assessment of Adsorption Free Energy for Peptide- Surface Interactions. Langmuir 2009, 25, 5637-5646. 